\title{
Synthetic sulfogalactosylceramide (sulfatide) and its use for the mass spectrometric quantitative urinary determination in metachromatic leukodystrophies
}

\author{
Yanli Cui • Benoit Colsch • Carlos Afonso • \\ Nicole Baumann • Jean-Claude Tabet • \\ Jean-Maurice Mallet $\cdot$ Yongmin Zhang
}

Published online: 19 September 2007

(C) Springer Science + Business Media, LLC 2007

\section{Erratum to: Glycoconj J}

DOI 10.1007/s10719-007-9067-7

The original version of this article unfortunately contained a mistake. The spelling of the third author's name was incorrect. Instead of "Carlos Alonso", it should be "Carlos Afonso".

The online version of the original article can be found at: http://dx.doi.org/10.1007/s10719-007-9067-7.

Y. Cui · J.-M. Mallet $\cdot$ Y. Zhang

Département de Chimie, Ecole Normale Supérieure,

UMR 8642: CNRS-ENS-UPMC,

24 rue Lhomond,

75231 Paris cedex 05, France

Y. Cui

Department of Chemistry, Zhejiang University,

Hangzhou 310027, China

Y. Zhang $(\bowtie)$

ZJU-ENS Joint Laboratory of Medicinal Chemistry,

Zhejiang University,

Hangzhou 310058, China

e-mail: yongmin.zhang@ens.fr

B. Colsch - C. Afonso - N. Baumann - J.-C. Tabet

Université Pierre et Marie Curie,

UMR CNRS 7613, 4 Place Jussieu,

75252 Paris cedex 05, France

B. Colsch $\cdot$ N. Baumann

Université Pierre et Marie Curie, UMR INSERM 711,

Hôpital de la Salpêtrière, 47 Bd de l'Hôpital,

75651 Paris cedex 13, France 